\title{
Evaluation of Critical Current Density of FeAs-based Superconductors
}

\author{
Edmund S. Otabe ${ }^{1 * *}$, Masaru Kiuchi ${ }^{1}$, Teruo Matsushita ${ }^{1}$, Baorong $\mathrm{Ni}^{2}$, Yanpeng $\mathrm{Qi}^{3}$, Lei Wang ${ }^{3}$, \\ Zhaoshun $\mathrm{Gao}^{3}$, Dongliang Wang ${ }^{3}$, Xianping Zhang ${ }^{3}$, Yanwei Ma ${ }^{3}$, Yasuyuki Nakajima ${ }^{4}$, Tsuyoshi Tamegai \\ ${ }^{1}$ Faculty of Computer Science and Systems Engineering, Kyushu Institute of Technology \\ 680 - 4 Kawazu, Iizuka, Fukuoka 820 - 8502, Japan \\ ${ }^{2}$ Department of Information Electronics, Fukuoka Institute of Technology, \\ 3-30-1 Wajiro-higashi, Higashi-ku, Fukuoka 811-0295, Japan \\ ${ }^{3}$ Institute of Electrical Engineering, Chinese Academy of Science, P. O. Box 2703, Beijing 100190, China \\ ${ }^{4}$ Department Applied Physics, The University of Tokyo, 7-3-1 Hongo, Bunkyo-ku, Tokyo 113-8656, Japan \\ ${ }^{5}$ TRIP-JST, Japan Science and Technology Agency, 7 Gobancho, Chiyoda-ku, Tokyo 102—0076, Japan
}

Received 5 April 2012; accepted 10 May 2012

\begin{abstract}
In this review paper, we report the characteristics of the critical current density in FeAs based superconductors which is newly discovered by Hosono group of Tokyo Institute of Technology on 2008. Since the many specimens in present stage are not single crystals, there are two kinds of critical current density observed in the specimens which are so-called local and global critical current densities. Therefore, it is necessary to evaluate both kinds of critical current densities. The history effect in which the global critical current density shows different values in increasing and decreasing magnetic field is also observed when the specimens have the local and the global critical current densities. The wire which critical current is $180 \mathrm{~A}$ is successfully developed with using the knowledge of abovementioned characteristics of two kinds of critical current densities and the history effect.
\end{abstract}

\section{INTRODUCTION}

FeP-based superconductor was found on 2006 by Kamihara et al. of Tokyo Institute of Technology [1]. Fe was believed not to be suitable to superconductivity. However, it was not attractive, since the critical temperature was only around $3 \mathrm{~K}$, and it is too low for practical applications. On February 2008, same group reported that the FeAs-based superconductor which critical temperature was $22 \mathrm{~K}$ [2]. Soon after, SmFeAsO (Sm-1111) was found by Chinese group on May 2008 and its critical temperature was $55 \mathrm{~K}$ [3]. Because of its high critical temperature, much more attentions were focused on FeAs-based superconductors. Many fundamental research works for practical application were performed such as superconducting wires [4-6], superconducting thin films[7-9], Josephson junctions [10, 11].

For engineering practical applications, it is essential to have enough high critical current density above $10^{10} \mathrm{~A} / \mathrm{m}^{2}$. However, since specimens are sintered bulk, there are two

* Corresponding author: otabe@cse.kyutech.ac.jp kinds of critical current density in the specimens. They are so-called local and global critical currents. That is, the connection between the grains in specimen is too week to carry large current, and the global critical current density is far lower than the local critical current density which flows only inside the grain. They are also called intra- and inter-grain shielding current density. It is essential to measure both local and global critical current densities, because the local critical current density will be achieved when the specimen will be prepared as single crystalline in near future which is already done in RE-123 (RE: Rare Earth) superconducting coated wire. Moreover, the global critical current is corresponding to transport critical current density which is usually measured by four probe method. In the present study, the local and the global critical current densities are measured by two measurements.

It is well known that the critical current density shows different values in increasing and decreasing magnetic field [12]. It is called history effect. The critical current density in decreasing magnetic field is higher than that in increasing magnetic field. This is caused by the existence of two kinds of the critical current densities. That is, the global critical current density which flows between the grains is affected by the magnetic field induced by the local critical current density which flows inside the grains. Then, the direction of the magnetic field changes according to increasing and decreasing magnetic field. In the present study, the history effect is used for evaluation of the local and the global critical current densities.

The critical current density is determined by the pinning strength. And the pinning strength is proportional to the product of the pinning efficiency, the size of pin, the density of the pin and the condensation energy density. For example, the value of the condensation energy density of $\mathrm{Nb}$-Ti is $2.2 \times 10^{5} \mathrm{~J} / \mathrm{m}^{3}$ at $0 \mathrm{~K}$ and is 5 times smaller than 
that of $\mathrm{Nb}_{3} \mathrm{Sn}$. Therefore, it is essential to improve the pinning efficiency to obtain high critical current density by introducing complicated form of $\alpha$-Ti as the pinning centers for practical $\mathrm{Nb}-\mathrm{Ti}$ wire. Therefore, it is necessary to estimate the condensation energy density of Fe-based superconductor, since the condensation energy density determined the performance of the critical current density of the material. In addition, the condensation energy density is a key to understand the future potential of the critical current density. In this report, the condensation energy density is estimated from the characteristics of the critical current density before and after the heavy ion irradiation which produces the artificial columnar shape pinning centers in the specimens.

Finally, the superconducting tape fabricated by PIT (Powder in Tube) method is introduced. The critical current reaches $180 \mathrm{~A}$ at $4.2 \mathrm{~K}$ in self-magnetic field.

\section{LOCAL AND GLOBAL CRITICAL CURRENT DENSITIES}

\subsection{Experiments and Analysis}

The samples were polycrystalline $\mathrm{Sr}_{0.6} \mathrm{~K}_{0.4} \mathrm{Fe}_{2} \mathrm{As}_{2}$ ((Sr,K)-122) superconductors prepared by a one-step solid state reaction method [13,14]. One was pure sample and other samples were prepared with addition of $\mathrm{Ag}$ of 10 and $20 \mathrm{wt} \%$. Table 1 shows the specifications of these samples. The typical sample size is $w=2 \mathrm{~mm}$ width, $l=3 \mathrm{~mm}$ length and $t=1 \mathrm{~mm}$ thickness.

The critical temperature, $T_{\mathrm{c}}$, was determined as the onset temperature of magnetic moment in field cool and zero field cool processes. The critical temperatures of the samples are almost the same as $34 \mathrm{~K}$. The microstructures of the samples were examined by scanning electron microscopy (SEM) [14]. The distributions of powder size in both samples were estimated by using so called line intercept method from SEM observations.

The local and global critical current densities are estimated by the observation result of remnant magnetic moment $m_{\mathrm{R}}$ at zero external magnetic field after an excursion of the magnetic field up to the maximum value $H_{\mathrm{m}}$ [15] . The external magnetic field is applied perpendicular to the wide surface of the sample. It is assumed that the observed remnant magnetic moment of the sample is composed of $m_{\mathrm{R} 1}$ for the current flowing uniformly through the slab sample and $m_{\mathrm{R} 2}$ for the current flowing in grains approximated by a sphere of radius $r$. The global shielding critical current density $J_{\text {cg }}$ is related to $m_{\mathrm{R} 1}$, and the local shielding critical current density $J_{\mathrm{cl}}$ related to $m_{\mathrm{R} 2}$. According to Bean's model, $H_{\mathrm{m}}$ dependence of $m_{\mathrm{R} 1}$ is

Table I

SPECIFICATIONS OF SAMPLES.

\begin{tabular}{llll}
\hline Sample & $T_{\mathrm{c}}[\mathrm{K}]$ & $\mu$ & $\sigma^{\prime}$ \\
\hline pure & 34.3 & 0.3 & 0.70 \\
10 wt.\% Ag & 34.4 & 1.0 & 0.55 \\
20 wt.\% Ag & 34.4 & 1.0 & 0.55 \\
\hline
\end{tabular}

given as

$$
\begin{aligned}
& m_{\mathrm{Rl}}= \\
& \left\{\begin{array}{lr}
\frac{w^{2} t H_{\mathrm{m}}^{2}}{4 H_{p 1}}\left(1+\frac{l}{w}-\frac{H_{m}}{H_{\mathrm{pl}}}\right) ; & 0<H_{m} \leq H_{p 1}, \\
\frac{w^{2} t H_{\mathrm{m}}^{3}}{12 H_{p 1}^{2}}\left[1-\frac{3 H_{p 1}}{H_{m}}\left(1+\frac{l}{w}\right)+\frac{12 l H_{p 1}^{2}}{w H_{m}^{2}}+\frac{2 H_{p 1}^{3}}{H_{m}^{3}}\left(1-\frac{3 l}{w}\right)\right] ; \\
H_{p 1}<H_{m}<2 H_{p 1}, \\
\frac{(3 l-w) w t H_{p 1}}{6} ; & H_{m}>2 H_{p 1}
\end{array}\right.
\end{aligned}
$$

where $H_{\mathrm{p} 1}=J_{\mathrm{cg}} w / 2$ is the penetration field of the slab sample. The derivative with respect to the maximum field $H_{\mathrm{m}}$ is given as

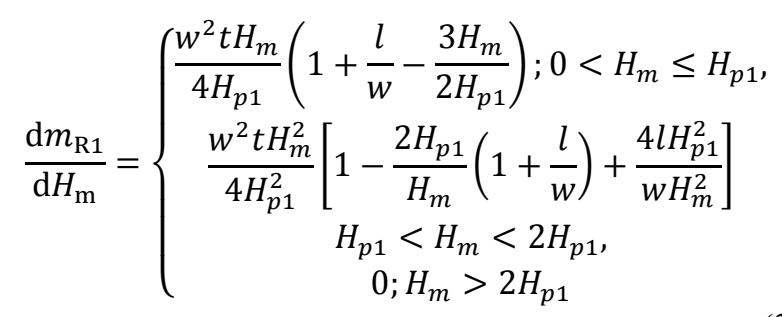

Since $\mathrm{d} m_{\mathrm{R} 1} / \mathrm{d} H_{\mathrm{m}}$ shows a peak at $H_{\mathrm{m}}=(1 / w+1) H_{\mathrm{p} 1} / 3$, the shielding current density through the whole size of samples, $J_{\text {cg }}$, can be estimated from the measured $H_{\mathrm{p} 1}$.

On the other hand, $m_{\mathrm{R} 2}$ is given as

$$
m_{R 2}=\left\{\begin{array}{c}
\frac{3 \pi^{2} r^{3} H_{m}^{2}}{8 H_{p 2}}\left(1-\frac{H_{m}}{H_{p 2}}+\frac{7 H_{m}^{2}}{24 H_{p 2}^{2}}\right) ; 0<H_{m} \leq H_{p 2} \\
\frac{\pi^{2} r^{3} H_{p 2}}{8}\left(-1+\frac{4 H_{m}}{H_{p 2}}-\frac{3 H_{m}^{2}}{H_{p 2}^{2}}+\frac{H_{m}^{3}}{H_{p 2}^{3}}-\frac{H_{m}^{4}}{8 H_{p 2}^{4}}\right) \\
H_{p 2}<H_{m}<2 H_{p 2} \\
\frac{\pi^{2} r^{3} H_{p 2}}{8} ; H_{m}>2 H_{p 2}
\end{array}\right.
$$

where $H_{\mathrm{p} 2}=J_{\mathrm{cl}} r$ is the penetration field of the grain. The derivative with respect to $H_{\mathrm{m}}$ is given as

$$
\begin{aligned}
& \frac{\mathrm{d} m_{R 2}}{\mathrm{~d} H_{m}} \\
& =\left\{\begin{array}{c}
\frac{3 \pi^{2} r^{3} H_{m}}{4 H_{p 2}}\left(1-\frac{3 H_{m}}{2 H_{p 2}}+\frac{7 H_{m}^{2}}{12 H_{p 2}^{2}}\right) ; 0<H_{m} \leq H_{p 2}, \\
\frac{\pi^{2} r^{3} H_{p 2}}{8}\left(\frac{4}{H_{p 2}}-\frac{6 H_{m}}{H_{p 2}^{2}}+\frac{3 H_{m}^{2}}{H_{p 2}^{3}}-\frac{H_{m}^{3}}{H_{p 2}^{4}}\right) \\
H_{p 2}<H_{m}<2 H_{p 2} \\
0 ; H_{-} m>2 H_{p 2}
\end{array}\right.
\end{aligned}
$$

In the above, $\mathrm{d} m_{\mathrm{R} 2} / \mathrm{d} H_{\mathrm{m}}$ shows a peak at $H_{\mathrm{m}}=$ $(6-2 \sqrt{2}) H_{p 2} / 7$. Therefore, $J_{\mathrm{cl}}$, intra-grain shielding current density, in the grain can be estimated from the measured $H_{\mathrm{p} 2}$. 
The whole magnetic moment of two kinds of currents $m_{\mathrm{R}}$ is given as

$$
m_{\mathrm{R}}=m_{\mathrm{R} 1}+m_{\mathrm{R} 2} N_{l}
$$

where $N_{1}$ is the number of grains in the sample. $N_{1}$ is approximately given by

$N_{\mathrm{l}}=\frac{V_{\mathrm{g}}}{V_{\mathrm{l}}} f=\frac{2 w l t f}{4 \pi r^{3}}$,

where $\mathrm{f}$ is the filling factor, $V_{\mathrm{g}}=w l t$ and $V_{\mathrm{l}}=4 \pi r^{3} / 3$ are volumes of slab sample and spherical grain, respectively. In this study, $m_{\mathrm{R}}$ was measured as a function of $H_{\mathrm{m}}$ using a SQUID magnetometer.

As the diameter of powder $D=2 r$ is distributed in polycrystalline samples, it is assumed that $D$ is statistically distributed, obeying the function:

$P(D)=\frac{1}{\sqrt{2 \pi} \sigma^{\prime} D} \exp \frac{-(\log D-\log \mu)^{2}}{2 \sigma^{\prime 2}}$,

where $\mu$ is the average size of the diameter of powder, and $\sigma^{\prime}$ is the standard deviation of the diameter of powder in logarithmic normal distribution. Then, the remnant magnetic moment $m_{\mathrm{R} 2}$ is given by

$m_{\mathrm{R} 2}^{\prime}\left(H_{m}\right)=\int_{0}^{\infty} m\left(D, H_{m}\right) P(D) \mathrm{d} D$.

The derivative $\mathrm{d} m{ }_{\mathrm{R} 2} / \mathrm{d} H_{\mathrm{m}}$ has a peak at some magnetic field $H_{\mathrm{mp}}$ as shown in Fig. 1 of Ref. [6], which is larger than the peak magnetic field $H_{\text {mp }}$. It means $J_{\mathrm{cl}}$ simply estimated from the average of the powder size using Eq. (4) is overestimated, and it is necessary to take account of the distribution of the powder size.

Fig. 1 shows the relationship between the derivative of the remnant magnetic moment and the external maximum magnetic field for Ag 20wt.\% added sample. Two peaks were clearly observed while only one peak was observed in pure sample. According to Eqs. (2) and (4) the peak of $\mathrm{d} m_{\mathrm{R}} / \mathrm{d} H_{\mathrm{m}}$, at smaller $H_{\mathrm{m}}$ is related to the global critical current density and the peak at larger $H_{\mathrm{m}}$ is related to the local critical current density. However, only one peak was clearly observed in pure sample. According to the value of the derivative and Eqs. (2) and (4), this peak is related to the local critical current density.

Fig. 2 shows $\sigma$ ' dependence of the ratio of the apparent local critical current density to the true local critical current density, $J_{\mathrm{c}}{ }^{\prime} / J_{\mathrm{c}}$. The $\sigma$ ' dependence of $J_{\mathrm{c}}{ }^{\prime} / J_{\mathrm{c}}$ is the same for $\mu$ in the range of $0.1-1.0 \mu \mathrm{m}$. Apparent $J_{\mathrm{c}}$ ' and true $J_{\mathrm{c}}$ is the same at $\sigma^{\prime}<0.1$. That is, the effect of the distribution of the powder size is small. On the contrary, $J_{\mathrm{c}}{ }^{\prime} / J_{\mathrm{c}}$ increases with increasing $\sigma^{\prime}$ for $\sigma^{\prime}>0.1$. The apparent $J_{\mathrm{c}}{ }^{\prime}$ is overestimated by ten times or more at about $\sigma^{\prime}=1$ and the effect of distribution of powder size becomes significant.

Fig. 3 shows the temperature dependence of the local and global critical current densities for various samples. The true local $J_{\mathrm{c}}$ estimated from the result of $J_{\mathrm{c}}{ }^{\prime} / J_{\mathrm{c}}$ in Fig. 2 is shown in Fig. 1. True $J_{c}$ is decreased by about three times by the Ag addition. The difference of local critical current density for two samples becomes small due to consideration of the effect of the distribution of the powder

\subsection{Results and Discussion}

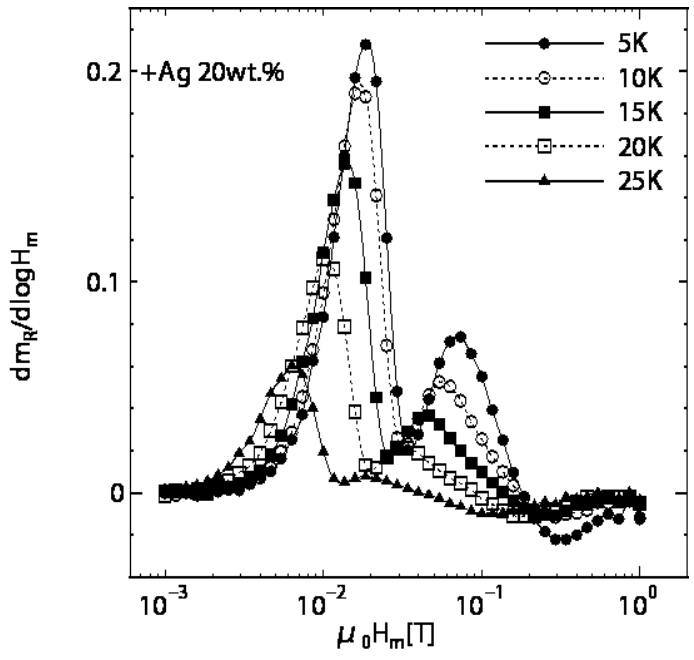

Fig. 1. Derivative of remnant magnetic moment as a function of maximum external magnetic field at various temperatures in sample with Ag 20wt.\% added.

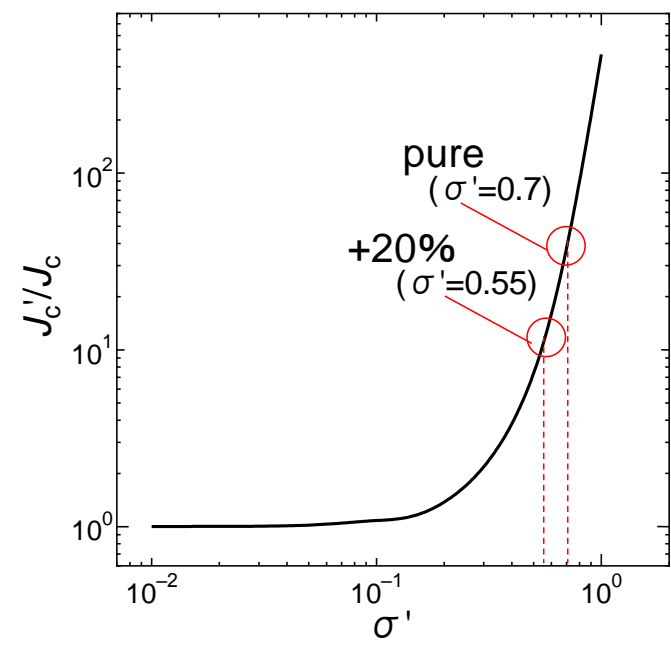

Fig. 2. Ratio of true and apparent critical current densities, $J_{\mathrm{c}}{ }^{\prime} / J_{\mathrm{c}}$, as a function of standard deviation $\sigma^{\prime}$.

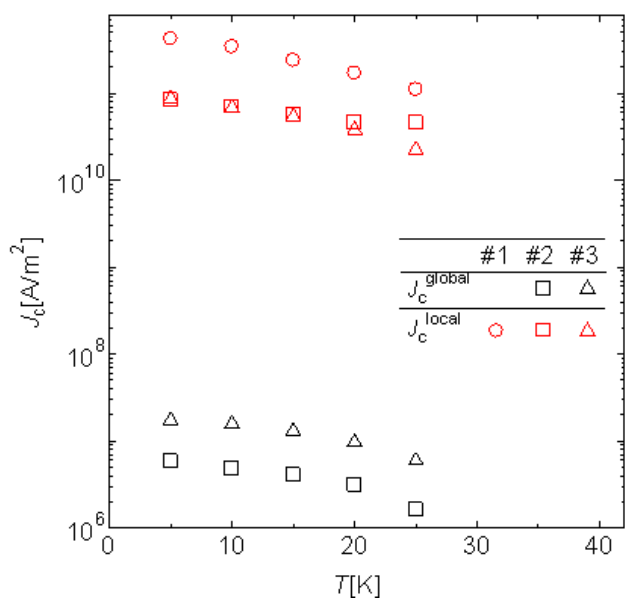

Fig. 3. Temperature dependence of the local and global critical current densities for sample \#1(pure), \#2(10wt.\% Ag added), \#3(20wt.\% Ag added). 
size. However, the reason for lower $J_{\mathrm{c}}$ in $\mathrm{Ag}$ addition is not clear. Since the effect of the distribution of powder size is large, it is important to take into account of the distribution of powder size on estimation of the local critical current density for $\sigma^{\prime}>0.1$. It is considered that the correction of local critical current density for the effect of distribution of powder size can be neglected for $\sigma^{\prime}<0.1$ which can be achieved by preparation of samples with small distribution of the powder size.

\section{HISTORY EFFECT IN CRITICAL CURRENT DENSITY}

\subsection{Experiments and Analysis}

In this section, two kinds of samples, i.e., $\mathrm{Sr}_{0.6} \mathrm{~K}_{0.4} \mathrm{Fe}_{2} \mathrm{As}_{2}+\mathrm{Ag}$ and $\mathrm{Sr}_{0.6} \mathrm{~K}_{0.4} \mathrm{Fe}_{2} \mathrm{As}_{2}+\mathrm{Pb}$, were prepared and the history effect in the critical current density was measured. The samples were formed into slabs with a typical size of $3.0 \mathrm{~mm}^{(w)} \times 0.6 \mathrm{~mm}^{(t)} \times 8.0 \mathrm{~mm}^{(l)}$. The critical temperature $T_{\mathrm{c}}$ obtained by ac susceptibility measurement was confirmed to be about $34 \mathrm{~K}$ for both samples.

The critical current densities of the bulk samples were measured and estimated by Campbell's method [16]. As one of the measurements dealing with the electromagnetic properties in superconductor, Campbell's method measures the penetrating ac flux in superconductor corresponding to the applied dc and variable ac magnetic fields. By analyzing the measured ac flux profile and ac magnetic field $b_{\text {ac }}$ vs. penetration depth $\lambda$ ' curve, one can derive not only the critical current density but also the relationship between the force on and the displacement of the flux lines. In this study, we focused our attention on the estimation of global and local $J_{\mathrm{c}}$ in bulk samples, i.e., only the critical current characteristics were derived and estimated. In the measurements, the temperature of

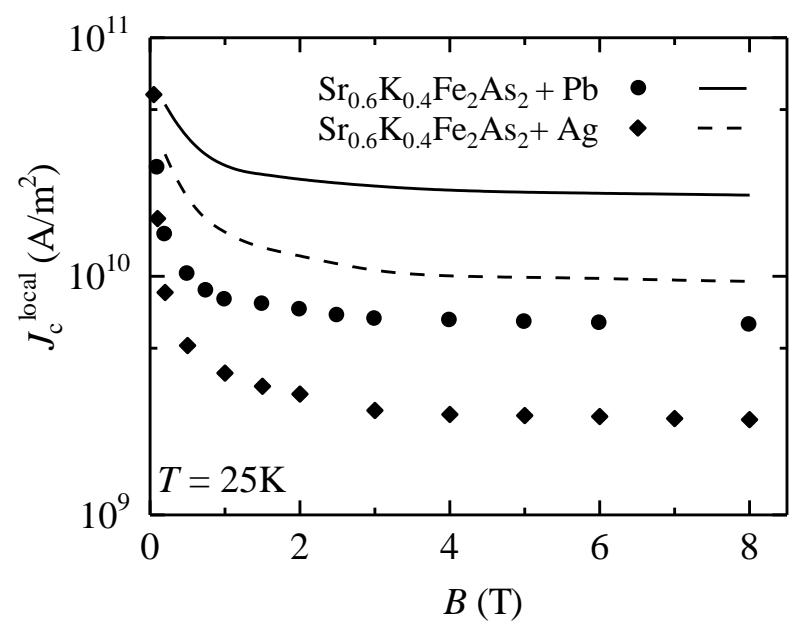

Fig. 4. Magnetic field dependence of local $J_{\mathrm{c}}$ in $(\mathrm{Sr}, \mathrm{K})-122$ samples with $10 \mathrm{wt} . \% \mathrm{~Pb}$ or $\mathrm{Ag}$ addition. The corrected curves in which the distribution of the grain size are shown by solid $((\mathrm{Sr}, \mathrm{K})-122+\mathrm{Pb})$ and dashed $((\mathrm{Sr}, \mathrm{K})-122+\mathrm{Ag})$ lines, respectively.

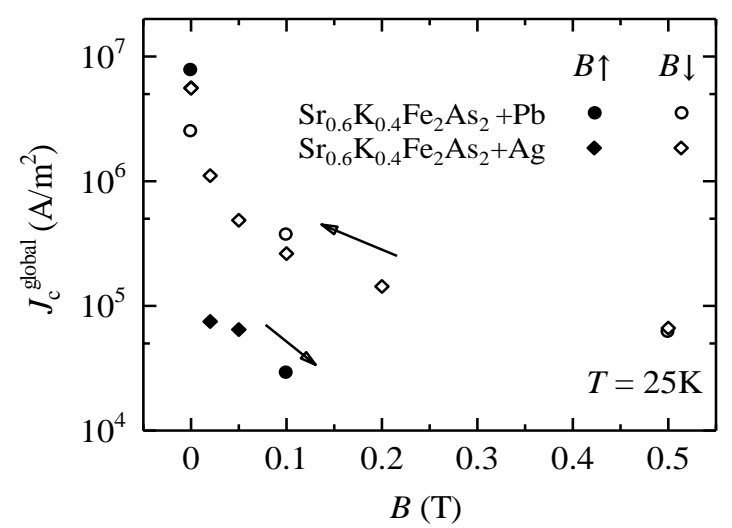

Fig. 5. Magnetic field dependence of global $J_{\mathrm{c}}$ in (Sr,K)-122 samples with $10 w t . \% \mathrm{~Pb}$ or $\mathrm{Ag}$ addition. The close and open symbols denote the processes of magnetic field increasing and decreasing, respectively.

samples was varied within the range of $18 \mathrm{~K}-35 \mathrm{~K}$, while the magnetic field was up to $8 \mathrm{~T}$. The frequency and the maximum amplitude of the ac magnetic field in Campbell's method were $97 \mathrm{~Hz}$ and $10 \mathrm{mT}$, respectively.

\subsection{Results and Discussion}

The magnetic field dependence of local critical current density for both samples is shown in Fig. 4. The value is in order of $10^{10} \mathrm{~A} / \mathrm{m}^{2}$ and is same to the result described in the previous section. That is, the local critical current density is measured by two kinds of measurement, and the results are the same.

Fig. 5 shows the magnetic field dependence of the critical current density in both samples. The close and open symbols denote the processes of dc magnetic field increasing and decreasing, respectively. As the data show clearly, unlike the local $J_{\mathrm{c}}$ characteristic, global $J_{\mathrm{c}}$ remains at an extremely poor property, and there is no apparent difference in global $J_{\mathrm{c}}$ between the samples with $\mathrm{Pb}$ and $\mathrm{Ag}$ additions. On the other hand, a significant history effect, i.e., global $J_{\mathrm{c}}$ takes a smaller value in the increasing dc magnetic field process than that in the decreasing one. Such a history effect could not be observed in local $J_{\mathrm{c}}$. This behavior has been also observed in some other superconductors [12, 17]. It was explained as a result caused from a superconducting micro-bridge structure, in which the super current flowing through the micro-bridge experiences a different magnetic field from the applied one, because of the demagnetizing field of the superconducting banks on each sides of the micro-bridge. In our case, the poor property of weak-link between the grains in superconducting polycrystalline ( $\mathrm{Sr}, \mathrm{K})-122$ can be regarded as the superconducting micro-bridge structures, and thus causes the significant history effect.

To investigate the history effect in detail, a variation of global $J_{\mathrm{c}}$ was measured after the sample experienced an excursion of magnetic field applying, i.e., applying a larger magnetic field $\left(B_{\mathrm{e}}\right)$ and then reducing it to a certain value 


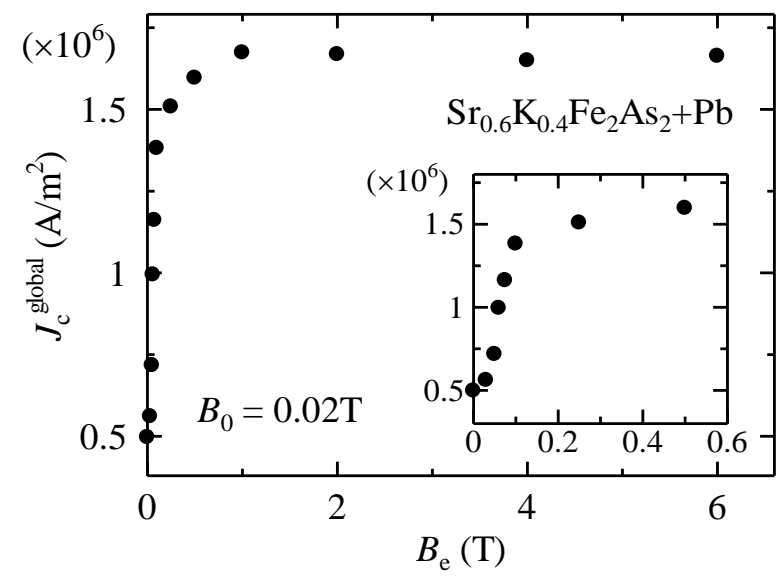

Fig. 6. Variation of global critical current density due to the magnetic field excursion at $B_{0}=0.02 \mathrm{~T}$.

$\left(B_{0}\right)$. Fig. 6 shows the experimental obtained result. Global $J_{\mathrm{c}}$ varies from a low value (corresponding to the increasing field process) to a high value (corresponding to the decreasing field process) under a field excursion no less than $B_{\mathrm{e}}=0.2 \mathrm{~T}$. According to the hypothesis on the history effect mentioned above and the basis of the critical state model, $B_{\mathrm{e}}$ approximately equals to $\mu 0 J_{\mathrm{cl}} g$, where $g$ is the grain size and $J_{\mathrm{cl}}$ is identical with the value of local $J_{\mathrm{c}}$ at $B_{0}=0.02 \mathrm{~T}$ and $T=25 \mathrm{~K}$. By using $g=2.5 \times 10^{-6} \mathrm{~m}$ and $B_{\mathrm{e}}$ $=0.2 \mathrm{~T}, J_{\mathrm{cl}}$ can be easily derived as to be $6.37 \times 10^{10} \mathrm{~A} / \mathrm{m}^{2}$, which turned out to be consistent with the value of $J_{\mathrm{cl}}$ shown in Fig. 4 which is measured by Campbell method.

\section{CONDENSATION ENERGY DENSITY}

\subsection{Experiments and Analysis}

Specimens were single crystalline $\mathrm{Ba}\left(\mathrm{Fe}_{1-x} \mathrm{Co}_{x}\right)_{2} \mathrm{As}_{2}$ grown by FeAs/CoAs self-flux method. The concentration of Co was determined by EDX measurements as $\mathrm{Ba}\left(\mathrm{Fe}_{0.93} \mathrm{Co}_{0.07}\right)_{2} \mathrm{As}_{2}$. The critical temperature was $T_{\mathrm{c}}=24$ $\mathrm{K}$ measured by the temperature dependence of magnetization in field-cooled and zero-field-cooled by a SQUID magnetometer. The heavy-ion irradiations of 200 $\mathrm{MeV} \mathrm{Au}$ was performed along $c$-axis using the TANDEM accelerator in JAEA. The heavy iron irradiations created columnar defects in the specimens. The matching magnetic field, $B_{\phi}$ is $2 \sim \mathrm{T}$ in all irradiations. The critical temperature does not change appreciably after the heavy-ion irradiations. The critical current density is estimated from the magnetic field dependence of the magnetization measured by a SQUID magnetometer. Further information is reported in references [18].

\subsection{Results and Discussion}

Fig. 7 shows the result of the magnetic field dependence of the critical current density of the Co doped Ba-122 sample before and after the Au heavy ion irradiation. The critical current density after the irradiation increases 6-7 times as large as that of before the irradiation from $2-20$ $\mathrm{K}$ at low magnetic fields. Hence, the columnar defects

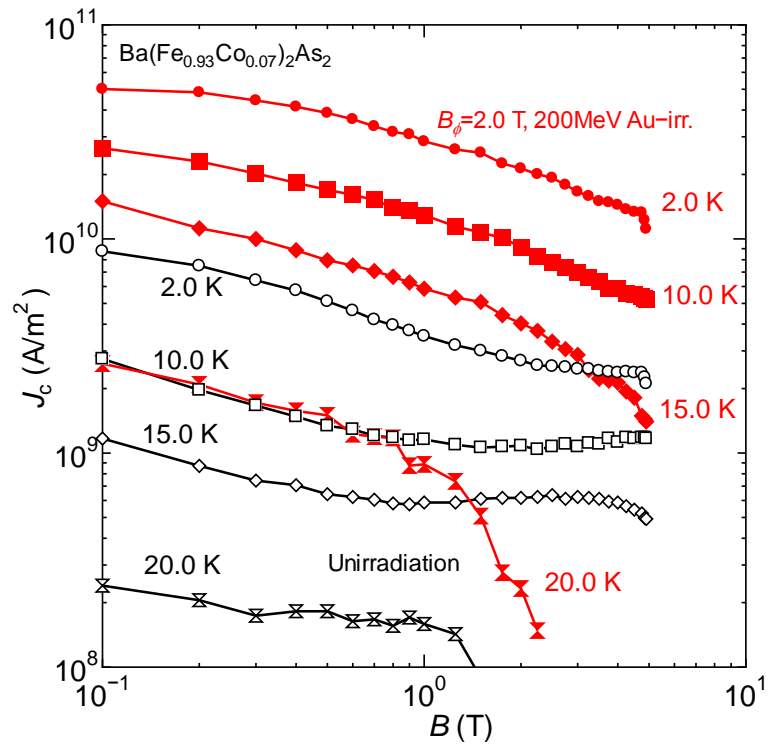

Fig. 7. Magnetic field dependence of $J_{c}$ in Co doped Ba-122 sample before (open symbol) and after (closed symbol) the Au heavy ion irradiation.

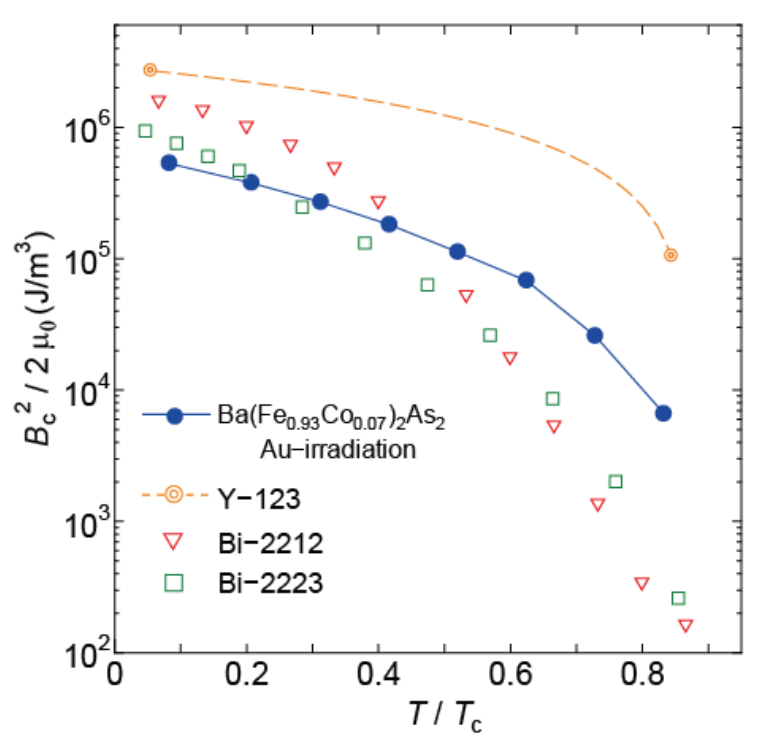

Fig. 8. Reduced temperature dependence of condensation energy density in various superconductors, Y-123 [20], Bi-2212, Bi-2223 [19].

are successfully introduced in the specimen and work as strong pinning centers. Then the condensation energy density is evaluated from the results of the critical current density. The further information for the theoretical calculation is described in reference [19].

Fig. 8 shows the temperature dependence of the condensation energy density of the sample and various superconductors $[19,20]$. The value at low temperatures is lower than those of cuprate superconductors, since the critical temperature and the upper critical field of Ba-122 are slightly smaller than those of the cuprate superconductors. However, the temperature dependence of the condensation energy density is close to that of Y-123. It is considered that the temperature dependence and the 
value of the condensation energy density are closely related to the anisotropy parameter, $\gamma_{\mathrm{a}}$, which is the ratio of the coherence length along $a-b$ plane and $c$-axis and reported as $\sim 2$ [21], while it is known as $\gamma_{\mathrm{a}} \sim 7$ for three dimensional Y-123. Hence, it is expected to use Ba-122 at high temperatures near $T_{\mathrm{c}}$ for future applications.

\section{DEVELOPMENT OF WIRE AND ENHANCEMENT OF CRITICAL CURRENT}

The world first wire of FeAs-based superconductor (La-1111) was reported on October 2008 [4]. Although, it was found that the wire showed the zero resistivity at around $20 \mathrm{~K}$, the superconducting transport current was not observed. However, superconducting shielding current was confirmed by the magnetic measurement. Therefore, the contact between the iron sheath and the superconductor is considered to be imperfect.

Wang et al. reported that the sheath material is crucial to obtain transport superconducting current in PIT (Powder in Tube) method for FeAs-based superconductor. They found that the $\mathrm{Ag}$ sheath is the best sheath material for the fabrication of high-performance tape and wire. Fig. 9 shows the magnetic field dependence of the critical current density for pure and $\mathrm{Ag}$ added $(\mathrm{Sr}, \mathrm{K})-122$ samples at $4.2 \mathrm{~K}$. Although the critical current density is in order of $10^{3}$ $\mathrm{A} / \mathrm{cm}^{2}$ and the critical current is only $9 \mathrm{~A}$, the critical current density is almost constant after rapid decrease at low magnetic field. The order of transport critical current density is same to the result of global critical current density as shown in Fig. 3. Therefore, the global critical current density is successfully estimated from the remnant magnetic moment method. The reason for the rapid decrease at low magnetic field is attributed to the weak link between the grains in the sample. Therefore, the history effect is also observed in the measurement of transport superconducting current. The critical current density is largely enhanced by addition of $\mathrm{Ag}$ to $(\mathrm{Sr}, \mathrm{K})-122$. This result is also consistent to the result shown in Fig. 3.

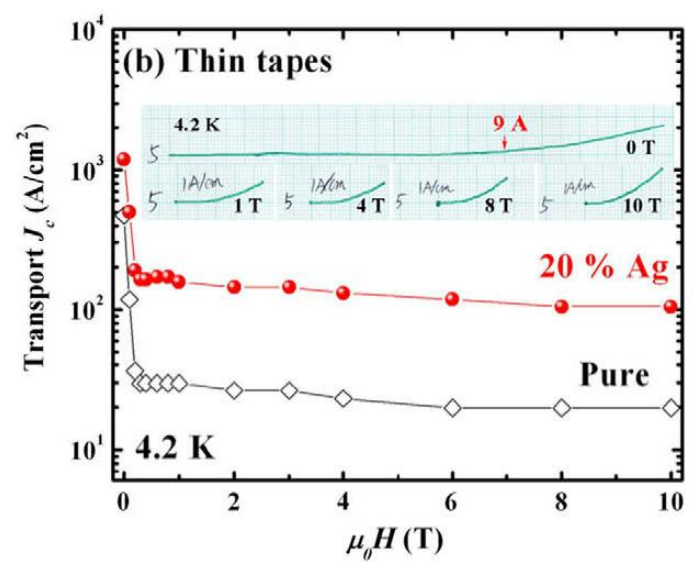

Fig. 9. Magnetic field dependence of transport critical current density for pure and $20 \mathrm{wt} . \% \mathrm{Ag}$ added samples at $4.2 \mathrm{~K}$. The critical current is $9 \mathrm{~A}$ at $4.2 \mathrm{~K}$ in self-magnetic field. [22].

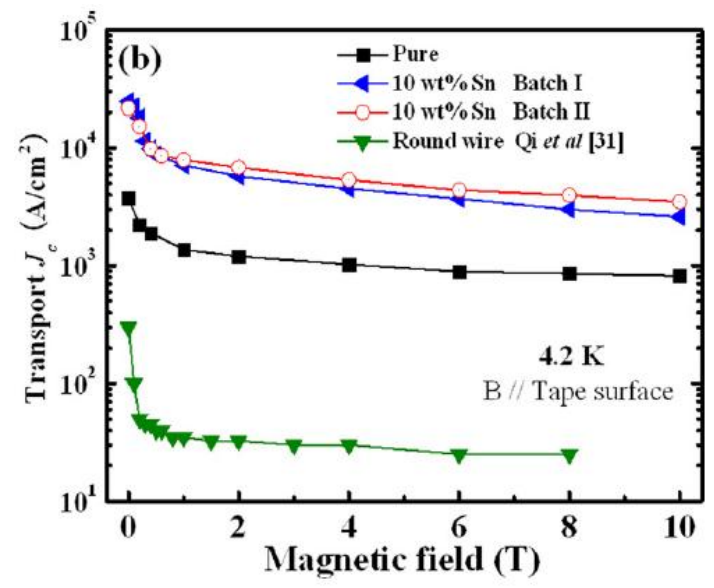

Fig. 10. Magnetic field dependence of transport critical current density for pure and $\mathrm{Sn}$ added $(\mathrm{Sr}, \mathrm{K})-122$ superconductors at $4.2 \mathrm{~K}$. The critical current is $180 \mathrm{~A}$ at $4.2 \mathrm{~K}$ in self-magnetic field. [25].

Togano et al. reported that large transport critical current densities observed in Ag-added $(\mathrm{Ba}, \mathrm{K}) \mathrm{Fe}_{2} \mathrm{As}_{2}$ $((\mathrm{Ba}, \mathrm{K})-122)$ superconducting wires prepared by an ex-situ PIT process [23]. The wire has a simple composite structure sheathed only by Ag. A precursor bulk material prepared by a melting process was ground into powder and put into a Ag tube. Transport critical current densities, $1.0 \times 10^{4} \mathrm{~A} / \mathrm{cm}^{2}$, critical current of $60.7 \mathrm{~A}$ in self-field are obtained. In $10 \mathrm{~T}, 1.1 \times 10^{3} \mathrm{~A} / \mathrm{cm}^{2}$, and $6.6 \mathrm{~A}$ are observed.

Moreover, Fujioka et al. reported ex-situ fabrication of superconducting $\mathrm{Sm}-1111$ wire by using a binder composed method [24]. The critical temperature is $36 \mathrm{~K}$ and the critical current density is about $4 \mathrm{kA} / \mathrm{cm}^{2}$ at $4.2 \mathrm{~K}$. The critical current is $30 \mathrm{~A}$. It is expected that the higher critical current will be achieved by optimizing the binder composition and/or amount in the sintering process.

Recently, Gao et al. reported the performance of grain alignment in $\mathrm{Sn}$-added $\mathrm{Sr}_{1-x} \mathrm{~K}_{x} \mathrm{Fe}_{2} \mathrm{As}_{2}$ superconducting tapes with Fe sheath prepared by ex-situ powder-in-tube method. At $4.2 \mathrm{~K}$, high transport critical current densities of $2.5 \times 10^{4} \mathrm{~A} / \mathrm{cm}^{2}$ (critical current of $180 \mathrm{~A}$ ) in self-field and $3.5 \times 10^{3} \mathrm{~A} / \mathrm{cm}^{2}$ (critical current 25.5 A) in $10 \mathrm{~T}$ have been measured as shown in Fig. 10.

Therefore, the critical current density and the critical current in FeAs-based superconductors increase very rapidly in these years. Further increase in the critical current density will be achieved by development of fabrication technique. It is important to estimate the correct value of the critical current density and to know the reason for the obstacles for the current flow.

\section{CONCLUSIONS}

In the present review paper, the characteristics of the critical current density in FeAs-based superconductors are estimated by several methods. And the results the two kinds of critical current densities, i.e., the local and global critical current densities are discussed. Although the local 
critical current density in the grains is in the order of $10^{11}$ $\mathrm{A} / \mathrm{m}^{2}$, the global critical current density though the grain is $10^{3}$ times smaller than the local critical current density. The history effect is observed where two kinds of the critical current densities exist. Therefore, it is useful to observe the history effect to estimate the local and global critical current densities for further development of the performance of transport current.

The condensation energy density which is directly connected to the characteristics of the critical current density is estimated from the result of critical current density before and after the heavy ion irradiation. The condensation energy density is enough high as cuprate superconductors and the temperature dependence is same to RE-123 superconductors. Therefore, the application near the critical temperature is expected.

Sn-added (Sr, K)-122 wire prepared by PIT method with Fe sheath and ex-situ powder. The critical current of 180 $A$ and critical current density of $2.5 \times 10^{4} \mathrm{~A} / \mathrm{cm}^{2}$ is obtained in self-field. The performance of critical current density is getting better and better, year by year. Therefore, further increase in the critical current density in FeAs-based superconductor is expected.

\section{REFERENCES}

[1] Y. Kamihara, H. Hiramatsu, M. Hirano, R. Kawamura, H. Yanagi, T. Kamiya, H. Hosono, "Iron-Based Layered Superconductor: LaOFeP”, J. Am. Chem. Soc. 128, pp. 10012-10013, 2006.

[2] Y. Kamihara, T. Watanabe, M. Hirano, H. Hosono, "Iron-Based Layered Superconductor $\mathrm{La}\left[\mathrm{O}_{1-x} \mathrm{~F}_{x}\right] \mathrm{FeAs}(x=0.05-0.12)$ with $T_{\mathrm{c}}=26 \mathrm{~K}$ ", J. Am. Chem. Soc. 130, pp. 3296-3297, 2008.

[3] Z.A. Ren, W. Lu, J. Yang, W. Yi, X.L. Shen, C. Zheng, G.C. Che, X.L. Dong, L.L. Sun, F. Zhou, Z.X. Zhao, "Superconductivity at 55 $\mathrm{K}$ in Iron-Based F-Doped Layered Quaternary Compound $\mathrm{Sm}\left[\mathrm{O}_{1-\mathrm{x}} \mathrm{F}_{\mathrm{x}}\right]$ FeAs", Chinese Phys. Lett. 25, pp. 2215, 2008.

[4] Z. Gao, L. Wang, Y. Qi, D. Wang, X. Zhang, Y. Ma, H. Yang, H. Wen, "Superconducting properties of granular $\mathrm{SmFeAsO}_{1-\mathrm{x}} \mathrm{F}_{\mathrm{x}}$ wires with $T_{\mathrm{c}}=52 \mathrm{~K}$ prepared by the powder-in-tube method", Supercond. Sci. Technol. 21 pp. 112001, 2008

[5] L. Wang, Y. Qi, D. Wang, Z. Gao, X. Zhang, Z. Zhang, C. Wang, Y $\mathrm{Ma}$, "Low-temperature synthesis of $\mathrm{SmFeAsO}_{0.7} \mathrm{~F}_{0.3-\delta}$ wires with a high transport critical current density”, Supercond. Sci. Technol. 27 pp. 075005, 2010.

[6] Z. Gao, L. Wang, C. Yao, Y. Qi, C. Wang, X. Zhang, D. Wang, C. Wang, Y. Ma, "High transport critical current densities in textured Fe-sheathed $\mathrm{Sr}_{1-\mathrm{x}} \mathrm{K}_{\mathrm{x}} \mathrm{Fe}_{2} \mathrm{As}_{2}+\mathrm{Sn}$ superconducting tapes", Appl. Phys. Lett., 99, pp. 242506-242509, 2011.

[7] H. Hiramatsu, T. Katase, T. Kamiya, M. Hirano, H. Hosono, "Superconductivity in Epitaxial Thin Films of Co-Doped $\mathrm{SrFe}_{2} \mathrm{As}_{2}$ with Bilayered FeAs Structures and their Magnetic Anisotropy", Appl. Phys. Express, 1, pp. 101702-101704, 2008.

[8] T. Kawaguchi, H. Uemura, T. Ohno, M. Tabuchi, T. Ujihara, K. Takenaka, Y. Takeda, H. Ikuta, "In situ growth of superconducting $\mathrm{NdFeAs}(\mathrm{O}, \mathrm{F})$ thin films by molecular beam epitaxy", Appl. Phys. Lett. 97, pp. 042509-042511, 2010.

[9] S. Haindl, M. Kidszun, A. Kauffmann, K. Nenkov, N. Kozlova, J. Freudenberger, T. Thersleff, J. Hänisch, J. Werner, E. Reich, L. Schultz, and B. Holzapfel, "High Upper Critical Fields and Evidence of Weak-Link Behavior in Superconducting $\mathrm{LaFeAsO}_{1-\mathrm{x}} \mathrm{F}_{\mathrm{x}}$ Thin Films" Phys. Rev. Lett. 104, pp. 077001-077004, 2010.
[10] T. Katase1, Y. Ishimaru, A. Tsukamoto, H. Hiramatsu, T. Kamiya, K. Tanabe, H. Hosono, "DC superconducting quantum interference devices fabricated using bicrystal grain boundary junctions in Co-doped $\mathrm{BaFe}_{2} \mathrm{As}_{2}$ epitaxial films", Supercond. Sci. Technol. 23 pp. 082001, 2010

[11] S. Schmidt, S. Döring, F. Schmidl, V. Grosse, P. Seidel, K. Iida, F. Kurth, S. Haindl, I. Mönch, B. Holzapfel, " $\mathrm{BaFe}_{1.8} \mathrm{Co}_{0.2} \mathrm{As}_{2}$ thin film hybrid Josephson junctions", Appl. Phys. Lett. 97, pp. $172504-172506,2010$.

[12] K. Watanabe, K. Noto, H. Morita, H. Fujimori, K. Mizuno, T. Aomine, B. Ni, T. Matsushita, K. Yamafuji and Y. Muto, "Anisotropy and hysteresis of transport critical currents in high temperature Ln-Y-Ba-Cu-O superconductors," Cryogenics 29 pp. $263-267,1989$

[13] Y. Ma, Z. Gao, L. Wang, Y. Qi, D. Wang, X. Zhang, "Simple One-Step Synthesis and Superconducting Properties of $\mathrm{SmFeAsO}_{1-x} \mathrm{~F}_{x}$ ", Chin. Phys. Lett. 26, 037401, 2009.

[14] L. Wang, Y. Qi, Z. Gao, D. Wang, X. Zhang, Y. Ma, "The role of silver addition on the structural and superconducting properties of polycrystalline $\mathrm{Sr}_{0.6} \mathrm{~K}_{0.4} \mathrm{Fe}_{2} \mathrm{As}_{2}$ ", Supercond. Sci. Technol. 23, $025027-025031,2010$

[15] A. Yamamoto, A.A. Polyanskii, J. Jiang, F. Kametani, C. Tarantini, F. Hunte, J.Jaroszynski, E.E. Hellstrom, P.J. Lee, A. Gurevich, D.C Larbalestier, Z.A. Ren, J. Yang, X.L. Dong, W. Lu, Z.X. Zhao, "Evidence for two distinct scales of current flow in polycrystalline Sm and Nd iron oxypnictides", Supercond. Sci. Technol., 21, 095008, 2008.

[16] A. M. Campbell, "The response of pinned flux vortices to low-frequency fields," J. Phys. C 2, pp. 1492-1501, 1969.

[17] T. Matsushita, B. Ni, K. Yamafuji, K. Watanabe, K. Noto, H Morita, H. Fujimori and Y. Muto, "History Dependence of Weakly Coupled Intergrain Currents in a Sintered Oxide Superconductor," Adv. Supercond. (Springer-Verlag, Tokyo, 1989) pp. 393-396.

[18] Y. Nakajima, Y. Tsuchiya, T. Taen, T. Tamegai, S. Okayasu, M. Sasase, "Enhancement of critical current density in Co-doped $\mathrm{BaFe} 2 \mathrm{~A} 2$ with columnar defects introduced by heavy-ion irradiation", Phys. Rev. B, 80, 012510(4pp), 2009.

[19] T. Matsushita M. Kiuchi, T. Haraguchi, T. Imada, K. Okamura, S Okayasu, S. Uchida, J. Shimoyama, K. Kishio, "Condensation energy density in Bi-2212 superconductors", Supercond. Sci. Technol. 19, pp. 200-205, 2006

[20] L. Civale, A.D. Marwick, T.K. Worthington, M.A. Kirk, J.R Thompson, L. Krusin-Elbaum, Y. Sun, J. R. Clem, F. Holtzberg, "Vortex confinement by columnar defects in $\mathrm{YBa} 2 \mathrm{Cu} 3 \mathrm{O} 7$ crystals: Enhanced pinning at high fields and temperatures", Phys. Rev. Lett. 67, pp. 648-651, 1991

[21] A. Yamamoto, J. Jaroszynski, C. Tarantini, L. Balicas, J. Jiang, A. Gurevich, D. C. Larbalestier, R. Jin, A. S. Sefat, M. A. McGuire, B.C. Sales, D. K. Christen, D. Mandrus, "Small anisotropy, weak thermal fluctuations, and high field superconductivity in Co-doped iron pnictide $\mathrm{Ba}\left(\mathrm{Fe}_{1-x} \mathrm{Co}_{x}\right)_{2} \mathrm{~A}_{2}$ ", Appl. Phys. Lett. 94, pp. 062511(3pp), 2009.

[22] L. Wang, Y. Qi, D. Wang, X. Zhang, Z. Gao, Z. Zhang, Y. Ma, S. Awaji, G. Nishijima, K.Watanabe, "Large transport critical currents of powder-in-tube $\mathrm{Sr}_{0.6} \mathrm{~K}_{0.4} \mathrm{Fe}_{2} \mathrm{As}_{2} / \mathrm{Ag}$ superconducting wires and tapes", Physica C, 470, pp.183-186, 2010

[23] K. Togano, A. Matsumoto, H. Kumakura, "Large Transport Critical Current Densities of Ag Sheathed (Ba,K)Fe2As2+Ag Superconducting Wires Fabricated by an Ex-situ Powder-in-Tube Process", Applied physics express 4(4), pp. 043101-043101-3, 2011.

[24] M. Fujioka, T.Kota, M. Matoba, T. Ozaki, Y. Takano, H. Kumakura, Y. Kamihara, "Effective Ex-situ Fabrication of F-Doped SmFeAsO Wire for High Transport Critical Current Density", Applied Physics Express 4, pp. 063102-063102-3, 2011.

[25] Z. Gao, L. Wang, C. Yao, Y. Qi, C. Wang, X. Zhang, D. Wang, C. Wang, Y. Ma, "High transport critical current densities in textured Fe-sheathed $\mathrm{Sr}_{1-\mathrm{x}} \mathrm{K}_{\mathrm{x}} \mathrm{Fe}_{2} \mathrm{As}_{2}+\mathrm{Sn}$ superconducting tapes", Applied Physics Letters, 99, pp. 242506-242506-3, 2011. 